Orthopäde 2009 · 38:1062

DOI 10.1007/s00132-009-1560-1

(C) Springer Medizin Verlag 2009

P. Kasten ${ }^{1}$. C.J.P. Simanski ${ }^{2} \cdot$ T. Volk $^{3} \cdot$ N. Schmelzer-Schmied ${ }^{1}$

${ }^{1}$ Stiftung Orthopädische Universitätsklinik, Universität Heidelberg

${ }^{2}$ Klinik für Unfallchirurgie, Orthopädie und Sporttraumatologie Köln-Merheim,

Lehrstuhl für Unfallchirurgie und Orthopädie der Universität Witten-Herdecke, Köln

${ }^{3}$ Klinik für Anästhesiologie und operative Intensivmedizin, Charité Campus

Mitte und Campus Virchow-Klinikum, Charité-Universitätsmedizin, Berlin

\title{
Erratum zu: Schmerztherapie in der Schulter-, Ellenbogen- und Handchirurgie
}

Der Orthopäde (2008) 37:970-976

Der Name des Zweitautors dieses Beitrags war in der online veröffentlichten Ausgabe der Zeitschrift fehlerhaft.

Wir bitten, dies zu entschuldigen und den korrigierten Namen zu berücksichtigen.

\section{Korrespondenzadresse}

\section{PD Dr. P. Kasten}

Stiftung Orthopädische

Universitätsklinik,

Universität Heidelberg

Schlierbacher Landstraße 200a,

69118 Heidelberg

Philip.Kasten@gmx.de

Die Online-Version des Originalartikels können Sie unter:

http://dx.doi.org/10.1007/s00132-008-1332-3 finden. 